\title{
High-energy dileptons from an anisotropic quark-gluon plasma
}

\author{
Mauricio Martinez ${ }^{\dagger}$ \\ Helmholtz Research School and Otto Stern School, Goethe-Universität Frankfurt am Main, \\ Germany \\ E-mail: guerrero@fias.uni-frankfurt.de
}

We calculate leading-order dilepton yields from a quark-gluon plasma which has a timedependent anisotropy in momentum space. Such anisotropies can arise during the earliest stages of quark-gluon plasma evolution due to the rapid longitudinal expansion of the created matter. A phenomenological model for the proper time dependence of the parton hard momentum scale, $p_{\text {hard }}$, and the plasma anisotropy parameter, $\xi$, is proposed. The model describes the transition of the plasma from a $0+1$ dimensional collisionally-broadened expansion at early times to a $0+1$ dimensional ideal hydrodynamic expansion at late times. We find that high-energy dilepton production is enhanced by pre-equilibrium emission up to $50 \%$ at LHC energies, if one assumes an isotropization/thermalization time of $2 \mathrm{fm} / \mathrm{c}$. Given sufficiently precise experimental data this enhancement could be used to determine the plasma isotropization time experimentally.

High-pT Physics at LHC -09

February 4- 42009

Prague, Czech Republic

\footnotetext{
* Speaker.

$\dagger$ The author gratefully acknowledges the collaboration and continuous support of Michael Strickland. This work was supported by the Helmholtz Research School and Otto Stern School of the Goethe-Universität Frankfurt am Main.
} 


\section{Introduction}

One of the most interesting problems facing the community in relativistic heavy ion collisions is to determine at what time the matter created can be described using hydrodynamics. In this context, at RHIC energies it has been found that for $p_{T} \lesssim 2 \mathrm{GeV}$, the elliptic flow of the matter created is described well by models which assume ideal hydrodynamic behavior starting at very early times $\tau \lesssim 1 \mathrm{fm} / \mathrm{c}[1,2,3,4]$. This is not completely understood due to the fact that the estimates from perturbative QCD for the thermalization time of a QGP at RHIC energies range from $2-3 \mathrm{fm} / \mathrm{c}[5,6,7]$. Moreover, recent results from conformal relativistic viscous hydrodynamics [8] have shown that these initial estimates for the isotropization/thermalization time of the plasma are not completely reliable due to poor knowledge of the proper initial conditions (CGC versus Glauber), details of plasma hadronization such as the choice of the proper freezeout time and the subsequent hadronic cascade, etc. Therefore, additional theoretical and experimental inputs are necessary to further constrain this time. It would be nice to have information about the thermalization time and appropiate initial conditions from other independent observables which are different than elliptic flow. One good candidate is high energy dileptons. Due to their large mean free path, lepton pairs can leave the strongly interacting medium after the collision. In this work, we examine the possibility to determine experimentally the thermalization time of the matter created using high energy dilepton yields as a function of both, mass and transverse momentum. We compute the expected $e^{+} e^{-}$yields resulting from a $\mathrm{Pb}-\mathrm{Pb}$ collision at LHC full beam energy, $\sqrt{s}=5.5 \mathrm{TeV}$ in a pre-equilibrium scenario of a quark-gluon plasma with a time-dependent anisotropy caused by the rapid longitudinal expansion. We find that at LHC energies, there is an enhancement of the dilepton yields as a function of the transverse momentum when $2<p_{T}<8 \mathrm{GeV}$ if one chooses isotropization/thermalization time of $2 \mathrm{fm} / \mathrm{c}$ [13].

This manuscript is organized as follows: In Sec. 1 we calculate the dilepton production rate at leading order using an anisotropic phase space distribution. In Sec. 2 we review the calculation of the dilepton yields from an anisotropic plasma. In Sec. 3 we discuss the main aspects of the interpolating model from collisionally-broadened to ideal hydrodynamical expansion. In Sec. 4 we present the expected medium dilepton yields for LHC energies. Finally, we present our conclusions and give an outlook in the Sec. 5 .

\section{Dilepton rate from kinetic theory}

From relativistic kinetic theory, the dilepton production rate $d N^{l^{+} l^{-}} / d^{4} X d^{4} P \equiv d R^{l^{+} l^{-}} / d^{4} P$ at leading order in the electromagnetic coupling, $\alpha$, is calculated as follows: $[9,10,11]$ :

$$
\frac{d R^{l^{+} l^{-}}}{d^{4} P}=\int \frac{d^{3} \mathbf{p}_{1}}{(2 \pi)^{3}} \frac{d^{3} \mathbf{p}_{2}}{(2 \pi)^{3}} f_{q}\left(\mathbf{p}_{1}\right) f_{\bar{q}}\left(\mathbf{p}_{2}\right) v_{q \bar{q}} \sigma_{q \bar{q}}^{l^{+} l^{-}} \delta^{(4)}\left(P-p_{1}-p_{2}\right)
$$

where $f_{q, \bar{q}}$ is the phase space distribution function of the medium quarks (anti-quarks), $v_{q \bar{q}}$ is the relative velocity between quark and anti-quark and $\sigma_{q \bar{q}}^{l^{+}} l^{-}$is the total cross section. Since we will be considering high-energy dilepton pairs with center-of-mass energies much greater than the dilepton mass we can ignore the finite lepton mass corrections and use simply $\sigma_{q \bar{q}}^{l^{+} l^{-}}=4 \pi \alpha^{2} / 3 M^{2}$. In addition, we assume that the distribution function of quarks and anti-quarks is the same, $f_{\bar{q}}=f_{q}$. 
We will follow the ansatz by Romatschke and Strickland for the distribution function that describes the pre-equilibrated stage of the plasma [12]. The ansatz consists in consider that any anisotropic distribution function is obtained from an arbitrary isotropic phase space distribution by squeezing $(\xi>0)$ or stretching $(\xi<0)$ the isotropic distribution function:

$$
f_{q, \bar{q}}\left(\mathbf{p}, \xi, p_{\text {hard }}\right)=f_{q, \bar{q}}^{i s o}\left(\sqrt{\mathbf{p}^{2}+\xi(\mathbf{p} \cdot \hat{\mathbf{n}})^{2}}, p_{\text {hard }}\right),
$$

where $p_{\text {hard }}$ is the hard momentum scale, $\hat{n}$ is the direction of the anisotropy and $\xi>0$ is a parameter that reflects the strength and type of anisotropy. In the isotropic case, $p_{\text {hard }}$ is identified with the temperature of the system and $\xi \equiv 0$. In this work we will study the case when the direction of the anisotropy is along the longitudinal (beamline) direction, i.e., $\hat{\mathbf{n}}=\hat{e}_{z}$. This configuration is relevant for earliest stages of the collisions between heavy nuclei.

From Eqs. (2.2) and (2.1) we obtain ${ }^{1}$ :

$$
\begin{aligned}
\frac{d R^{l^{+} l^{-}}}{d^{4} P} & =\frac{5 \alpha^{2}}{18 \pi^{5}} \int_{-1}^{1} d\left(\cos \theta_{p_{1}}\right) \int_{a_{+}}^{a_{-}} \frac{d p_{1}}{\sqrt{\chi}} p_{1} f_{q}\left(\sqrt{\mathbf{p}_{\mathbf{1}}^{2}\left(\mathbf{1}+\xi(\tau) \cos ^{2} \theta_{\mathbf{p}_{\mathbf{1}}}\right)}, p_{\text {hard }}(\tau, \eta)\right) \\
& \times f_{\bar{q}}\left(\sqrt{\left(\mathbf{E}-\mathbf{p}_{\mathbf{1}}\right)^{\mathbf{2}}+\xi(\tau)\left(\mathbf{p}_{\mathbf{1}} \cos \theta_{\mathbf{p}_{\mathbf{1}}}-\mathbf{P} \cos \theta_{\mathbf{P}}\right)^{\mathbf{2}}}, p_{\text {hard }}(\tau, \eta)\right)
\end{aligned}
$$

with

$$
\begin{aligned}
\chi & =4 P^{2} p_{1}^{2} \sin ^{2} \theta_{P} \sin ^{2} \theta_{p_{1}}-\left(2 p_{1}\left(E-P \cos \theta_{P} \cos \theta_{p_{1}}\right)-M^{2}\right)^{2} \\
a_{ \pm} & =\frac{M^{2}}{2\left(E-P \cos \left(\theta_{P} \pm \theta_{p_{1}}\right)\right)} .
\end{aligned}
$$

Notice that the dilepton rate does not tell us anything about the space-time evolution of the system and, therefore, it is not enough to make a phenomenological prediction for the expected dilepton yields. In order to make contact with experiments, it is necessary to include the space-time dependence of $p_{\text {hard }}$ and $\xi$ and then integrate over the space-time volume

$$
\begin{aligned}
& \frac{d N^{l^{+} l^{-}}}{d M^{2} d y}=\pi R_{T}^{2} \int d^{2} p_{T} \int_{\tau_{0}}^{\tau_{f}} \int_{-\infty}^{\infty} \frac{d R^{l^{+} l^{-}}}{d^{4} P} \tau d \tau d \eta, \\
& \frac{d N^{l^{+} l^{-}}}{d^{2} p_{T} d y}=\pi R_{T}^{2} \int d M^{2} \int_{\tau_{0}}^{\tau_{f}} \int_{-\infty}^{\infty} \frac{d R^{l^{+} l^{-}}}{d^{4} P} \tau d \tau d \eta,
\end{aligned}
$$

where $R_{T}=1.2 A^{1 / 3} \mathrm{fm}$ is the radius of the nucleus in the transverse plane. These expressions are evaluated in the center-of-mass (CM) frame while the dilepton production rate is calculated for the local rest frame (LR) of the emitting region. Then, the dilepton pair energy has to be understood as $E_{L R}=p_{T} \cosh (y-\eta)$ in the differential dilepton rate $d R_{\mathrm{ann}} / d^{4} P$. Substituting Eq. (2.3) into Eqs. (2.4), we obtain the dilepton spectrum including the effect of a time-dependent momentum anisotropy. In Eqs. (2.4) it is assumed that there is only longitudinal expansion of the system. This assumption is well justified since corrections to high energy dileptons coming from transverse expansion or mixed/hadronic phase do not play an important role in the studied kinematic regime [14].

${ }^{1}$ Details of the calculation are presented in Ref. [13]. 
Note that we have not included the next-to-leading order (NLO) corrections to the dilepton rate due to the complexity of these contributions for finite $\xi$. These affect dilepton production for isotropic systems for $E / T \lesssim 1[15,16,17,18]$. In the regions of phase space where there are large NLO corrections, we will apply $K$-factors to our results as indicated.

\section{Space-time model}

Previous phenomenological studies of high energy dileptons have assumed that the value of the isotropization time, $\tau_{\text {iso }}$, is of the same order as the parton formation time $\tau_{0}$. However, the physical mechanisms which could make such fast isotropization feasible are not completely understood. Recently, a phenomenological model has been proposed where the isotropization time could be larger as a consequence of a previous evolution in a pre-equilibrium dynamics [13, 14]. Before going into the details of the model, we remind the reader of some general relations.

First, the plasma anisotropy parameter $\xi$ is related with the average longitudinal momentum $\left(p_{L}\right)$ and transverse momentum $\left(p_{T}\right)$ of the hard particles through the expression:

$$
\xi=\frac{\left\langle p_{T}^{2}\right\rangle}{2\left\langle p_{L}^{2}\right\rangle}-1
$$

Note that this relation is completely general and can be applied in all cases. It is possible to obtain two important limits from Eq. (3.1). When we have that $\left\langle p_{T}^{2}\right\rangle=2\left\langle p_{L}^{2}\right\rangle$, then $\xi=0$. This is the case when the system is isotropic in momentum-space. Another possibility is when the partons expand freely along the longitudinal axis, i.e., 1d free streaming expansion. Using the free streaming distribution function, it is possible to show that the transverse and longitudinal momentum scales as $[5,13,19]$ :

$$
\begin{aligned}
& \left\langle p_{T}^{2}\right\rangle_{\text {f.s. }} \propto 2 T_{0}^{2}, \\
& \left\langle p_{L}^{2}\right\rangle_{\text {f.s. }} \propto T_{0}^{2} \frac{\tau_{0}^{2}}{\tau^{2}} .
\end{aligned}
$$

Inserting these expressions into Eq. (3.1), one obtains $\xi_{f . s .}(\tau)=\tau^{2} / \tau_{0}^{2}-1$. The free streaming result for $\xi$ is the upper-bound on possible momentum-space anisotropies developed during $1 \mathrm{~d}$ expansion by causality. When the system has different kind of interactions, Eq. (3.1) for the anisotropy parameter $\xi$ will scale differently as discussed below.

Second, for a given anisotropic phase space distribution of the form specified in Eq. (2.2), the local energy density can be factorized as:

$$
\begin{aligned}
\mathscr{E}\left(p_{\text {hard }}, \xi\right) & =\int \frac{d^{3} \mathbf{p}}{(2 \pi)^{3}} p f_{\text {iso }}\left(\sqrt{\mathbf{p}^{2}+\xi(\mathbf{p} \cdot \hat{\mathbf{n}})^{2}}, p_{\text {hard }}\right), \\
& =\mathscr{E}_{0}\left(p_{\text {hard }}\right) \mathscr{R}(\xi)
\end{aligned}
$$

where $\mathscr{E}_{0}$ is the initial local energy density deposited in the medium at $\tau_{0}$ and

$$
\mathscr{R}(\xi) \equiv \frac{1}{2}\left(\frac{1}{1+\xi}+\frac{\arctan \sqrt{\xi}}{\sqrt{\xi}}\right) .
$$

In this work, we will not study explicitly the possibility of $1 \mathrm{~d}$ free streaming expansion since, in reality, this is a rather extreme assumption which requires that the partons do not interact at all [13]. 


\subsection{Momentum-space broadening and plasma instabilities effect}

The ratio between the average longitudinal and transverse momentum needed to compute $\xi$ using Eq. (3.1) is modified from the free streaming case if collisions between the partons are taken into account. In general, it is a difficult task to obtain the exact form of collisional kernel of the Boltzmann equation plus mean field interactions (Vlasov term). As a first approach, one can start considering the effect of elastic collisions in the broadening of the longitudinal momentum of the particles. This was the approach in the original version of the bottom-up scenario [5]. In the first stage of this scenario, $1 \ll Q_{s} \tau \ll \alpha_{s}^{3 / 2}$, initial hard gluons have typical momentum of order $Q_{s}$ and occupation number of order $1 / \alpha_{s}$. Due to the fact that the system is expanding at the speed of light in the longitudinal direction, the density of hard gluons decreases with time, $N_{g} \sim Q_{s}^{3} /\left(\alpha_{s} Q_{s} \tau\right)$. If there were no interactions this expansion would be equivalent to $1+1$ free streaming and the longitudinal momentum $p_{L}$ would scale like $1 / \tau$. However, once elastic $2 \leftrightarrow 2$ collisions of hard gluons are taken into account, the ratio between the longitudinal momentum $p_{L}$ and the typical transverse momentum of a hard particle $p_{T}$ decreases as [5]:

$$
\frac{\left\langle p_{L}^{2}\right\rangle}{\left\langle p_{T}^{2}\right\rangle} \propto\left(Q_{s} \tau\right)^{-2 / 3}
$$

Assuming, as before, isotropy at the formation time, $\tau_{0}=Q_{s}^{-1}$, this implies that for a collisionallybroadened expansion, $\xi(\tau)=\left(\tau / \tau_{0}\right)^{2 / 3}-1$.

Note that in order to obtain the relation given by Eq. (3.5) it is implicitly assumed that that the elastic cross-section is screened at long distances by an isotropic real-valued Debye mass [5]. But this is not the case of an anisotropic plasma, since the Debye mass can become complex due to the chromo-Weibel instability $[12,19,20,21,22]$. Such negative eigenvalues indicate instabilities, which result in exponential growth of chromo-electric and magnetic fields, $E^{a}$ and $B^{a}$, respectively. These fields deflect the particles and how much deflection occurs will depend on the amplitude and domain size of the induced chromofields. Currently, the precise parametric relation between the plasma anisotropy parameter and the amplitude and domain size of the chromofields is not known from first principles.

If one would like to include the momentum-space broadening and the impact of the plasma instabilities, this can be achieved by considering the temporal dependence of $\xi(\tau)$ as:

$$
\xi(\tau, \delta)=\left(\frac{\tau}{\tau_{0}}\right)^{\delta}-1
$$

The exponent $\delta$ contains the physical information about the particular type of momentum-space broadening which occurs due to plasma interactions. Two limiting cases for this exponent are the ideal hydrodynamic and free streaming expansion. In the $1+1$ hydrodynamical limit, $\delta \equiv 0$ and then $\xi \rightarrow 0$. For $\delta \equiv 2$, one recovers the $1+1$ dimensional free streaming case, $\xi \rightarrow \xi_{\text {f.s. }}=\left(\tau / \tau_{0}\right)^{2}-1$. For $0<\delta<2$, one obtains the proper-time dependence of the energy density and temperature by substituting (3.6) into the general expression for the factorized energy density (3.3) to obtain $\mathscr{E}(\tau, \delta)=\mathscr{E}_{0}\left(p_{\text {hard }}\right) \mathscr{R}(\xi(\tau, \delta))$.

Different values of $\delta$ arise dynamically from the different processes contributing to parton isotropization. For example, elastic collisional-broadening results in Eq. (3.5) and hence $\delta=2 / 3$. 
Recently, some authors have considered the values of $\delta$ resulting from processes associated with the chromo-Weibel instability presented at the earliest times after the initial nuclear impact [23, 24, 25]:

$$
\frac{\left\langle p_{L}^{2}\right\rangle}{\left\langle p_{T}^{2}\right\rangle} \sim\left(Q_{s} \tau\right)^{-\frac{1}{2}\left(\frac{1}{1+v}\right)}
$$

where

$$
v= \begin{cases}0 & \text { Ref.[23] } \\ 1 & \text { Ref.[24] } \\ 2 & \text { Nielsen-Olesen limit, Ref.[25] }\end{cases}
$$

Summarizing, the coefficient $\delta$ in Eq. (3.6) takes on the following values

$$
\delta=\left\{\begin{aligned}
2 & \text { Free streaming expansion } \\
2 / 3 & \text { Collisional-Broadening, Ref.[5] } \\
1 / 2 & \text { Ref.[23] } \\
1 / 4 & \text { Ref.[24] } \\
1 / 6 & \text { Nielsen-Olesen limit, Ref.[25] } \\
0 & \text { Hydrodynamic expansion }
\end{aligned}\right.
$$

In Fig. 1 we sketch the time-dependence of the plasma anisotropy parameter indicating the time scales at which the various processes become important. At times shorter than the mean time between successive elastic scatterings, $\tau_{\mathrm{MFP}}$, the system will undergo $1+1$ dimensional free streaming with $\delta=2$. For times long compared to $\tau_{\mathrm{MFP}}$ but short compared to $\tau_{\text {Instability }}$ the plasma anisotropy will grow with the collisionally-broadened exponent of $\delta=2 / 3$. Here $\tau_{\text {Instability }}$ is the time at which instability-induced soft gauge fields begin to influence the hard-particles' motion. When $\tau_{\text {Instability }}<\tau<\tau_{\text {iso }}$ the plasma anisotropy grows with the slower exponent of $\delta=1 / 6 \ldots 1 / 2$ due to the bending of particle trajectories in the induced soft-field background. At times large compared to $\tau_{\text {Instability }}$ inelastic processes are expected to drive the system back to isotropy [5]. We note here that for small $\xi$ and realistic couplings it has been shown [26] that one cannot ignore the effect of collisional-broadening of the distribution functions and that this may completely eliminate unstable modes from the spectrum.

From this sketch, it may be possible to try to construct a detailed model which includes all of the various time scales and study the dependence of the process under consideration on each. However, there are theoretical uncertainties in each of these time scales and their dependences on experimental conditions. Because of this, we will construct a phenomenological model which smoothly interpolates the coefficient $\delta$ from the $1 \mathrm{~d}$ collisionally-broadened expansion to $1 \mathrm{~d}$ hydrodynamical expansion, i.e., $2 / 3 \geq \delta \geq 0$.

In the model we introduce a transition width, $\gamma^{-1}$, which indicates the smoothness of the tran-

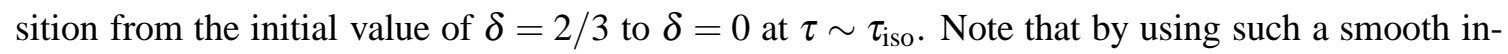
terpolation one can achieve a reasonable phenomenological description of the transition from nonequilibrium to equilibrium dynamics which should hopefully capture the essence of the physics. The collisionally-broadened interpolating model provides us a realistic estimate of the effect of plasma anisotropies. 


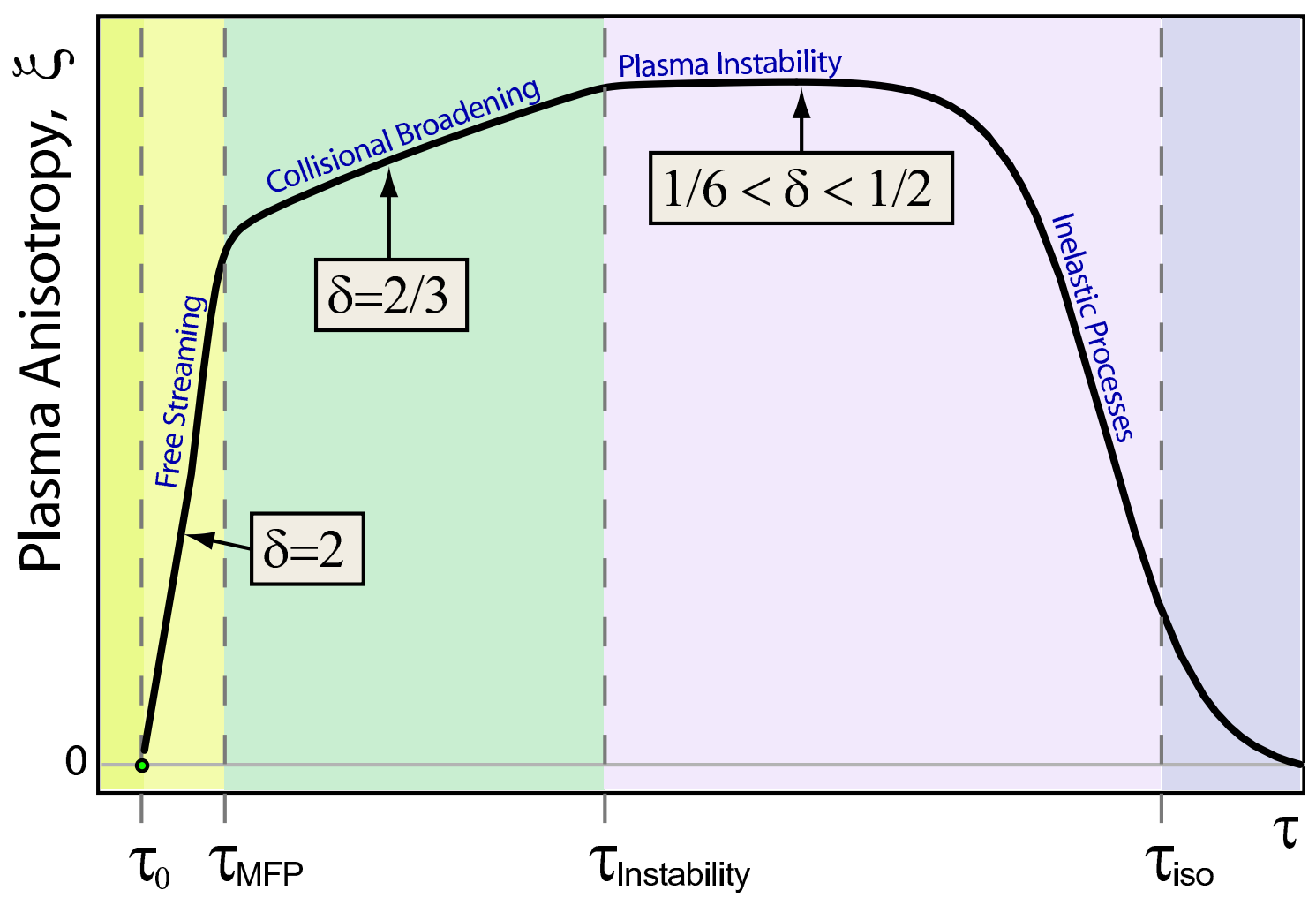

Figure 1: Sketch of the time dependence of the plasma anisotropy indicating the various time scales and processes taking place. Here $\tau_{\text {MFP }}$ is the mean time between elastic collisions (mean-free time) and $\tau_{\text {Instability }}$ is the time at which plasma-instability induced soft modes have grown large enough to affect hard particle dynamics.

\subsection{Interpolating model for collisionally broadened expansion}

In order to construct an interpolating model between collisionally-broadened and hydrodynamical expansion, we introduce the smeared step function:

$$
\lambda\left(\tau, \tau_{\text {iso }}, \gamma\right) \equiv \frac{1}{2}\left(\tanh \left[\frac{\gamma\left(\tau-\tau_{\text {iso }}\right)}{\tau_{\text {iso }}}\right]+1\right)
$$

where $\gamma^{-1}$ sets the width of the transition between non-equilibrium and hydrodynamical evolution in units of $\tau_{\text {iso. }}$. In the limit when $\tau \ll \tau_{\text {iso }}$, we have $\lambda \rightarrow 0$ and when $\tau \gg \tau_{\text {iso }}$ we have $\lambda \rightarrow 1$. Physically, the energy density $\mathscr{E}$ should be continuous as we change from the initial non-equilibrium value of $\delta$ to the final isotropic $\delta=0$ value appropriate for ideal hydrodynamic expansion. Once the energy density is specified, this gives us the time dependence of the hard momentum scale. We find that for general $\delta$ this can be accomplished with the following model for fixed final multiplicity [13]:

$$
\begin{aligned}
\xi(\tau, \delta) & =\left(\tau / \tau_{0}\right)^{\delta(1-\lambda(\tau))}-1, \\
\mathscr{E}(\tau, \eta) & =\mathscr{E}_{0} \mathscr{R}(\xi) \overline{\mathscr{U}}^{4 / 3}(\tau), \\
p_{\text {hard }}(\tau, \eta) & =T_{0} \overline{\mathscr{U}}^{1 / 3}(\tau),
\end{aligned}
$$


with $\mathscr{R}(\xi)$ defined in Eq. (3.4) and for fixed final multiplicity we have:

$$
\begin{aligned}
\overline{\mathscr{U}}(\tau) & \equiv \mathscr{U}(\tau) / \mathscr{U}\left(\tau_{\text {iso }}^{+}\right), \\
\mathscr{U}(\tau) & \equiv\left[\mathscr{R}\left(\left(\tau_{\text {iso }} / \tau_{0}\right)^{\delta}-1\right)\right]^{3 \lambda(\tau) / 4}\left(\frac{\tau_{\text {iso }}}{\tau}\right)^{1-\delta(1-\lambda(\tau)) / 2}, \\
\mathscr{U}\left(\tau_{\text {iso }}^{+}\right) & \equiv \lim _{\tau \rightarrow \tau_{\text {iso }}^{+}} \mathscr{U}(\tau)=\left[\mathscr{R}\left(\left(\tau_{\text {iso }} / \tau_{0}\right)^{\delta}-1\right)\right]^{3 / 4}\left(\frac{\tau_{\text {iso }}}{\tau_{0}}\right) .
\end{aligned}
$$

and $\delta=2 / 3$ for the case of $1 \mathrm{~d}$ collisionally broadened expansion interpolating to $1 \mathrm{~d}$ ideal hydrodynamic expansion.
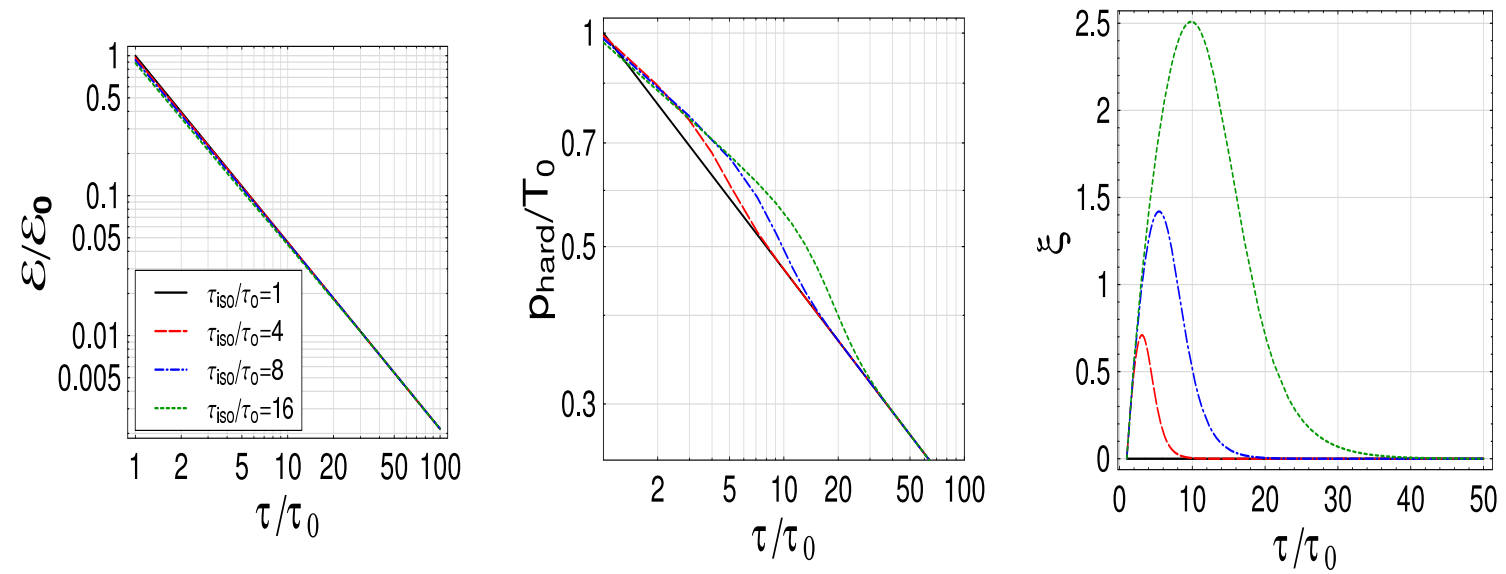

Figure 2: Temporal evolution using our fixed final multiplicity interpolating models for the energy density (left column), hard momentum scale (middle column), and anisotropy parameter (right column) for four different isotropization times $\tau_{\text {iso }} \in\{1,4,6,18\} \tau_{0}$. To convert to physical scales use $\tau_{0} \sim 0.3 \mathrm{fm} / \mathrm{c}$ for RHIC and $\tau_{0} \sim 0.1 \mathrm{fm} / \mathrm{c}$ for LHC.

In Fig. 2, the temporal evolution of the energy density, hard momentum scale and the anisotropy parameter $\xi(\tau)$ is plotted using Eq. (3.11a). As can be seen from the figure for fixed final multiplicity at $\tau=\tau_{0}$, the initial value of $p_{\text {hard }}$ is reduced for finite values of $\tau_{\text {iso }}$ but once the system looks isotropic in momentum-space around $\tau \gtrsim \tau_{\text {iso }}, p_{\text {hard }}$ is the same, independent of its initial value [13].

\section{Results}

We calculate predicted $e^{+} e^{-}$yields as a function of invariant mass and transverse momentum along with predicted yields from other sources for LHC energies. For comparison with previous works we take $\tau_{0}=0.088 \mathrm{fm} / \mathrm{c}, T_{0}=845 \mathrm{MeV}, T_{c}=160 \mathrm{MeV}$, and $R_{T}=7.1 \mathrm{fm} \mathrm{[18].} \mathrm{Here} \mathrm{we}$ assume that when the system reaches $T_{c}$ all medium emission stops.

In Fig. 3 we show our predicted dilepton mass spectrum for LHC energies using the time dependence of the energy density, the hard momentum scale and the anisotropy parameter given by Eqns. (3.11) with $\delta=2 / 3$. As can be seen from Fig. 3 there is a difference of the medium dilepton yield when varying the assumed plasma isotropization time from $0.088 \mathrm{fm} / \mathrm{c}$ to $2 \mathrm{fm} / \mathrm{c}$. However, the prediction is up to one order of magnitude below the other contributions of dilepton yields 


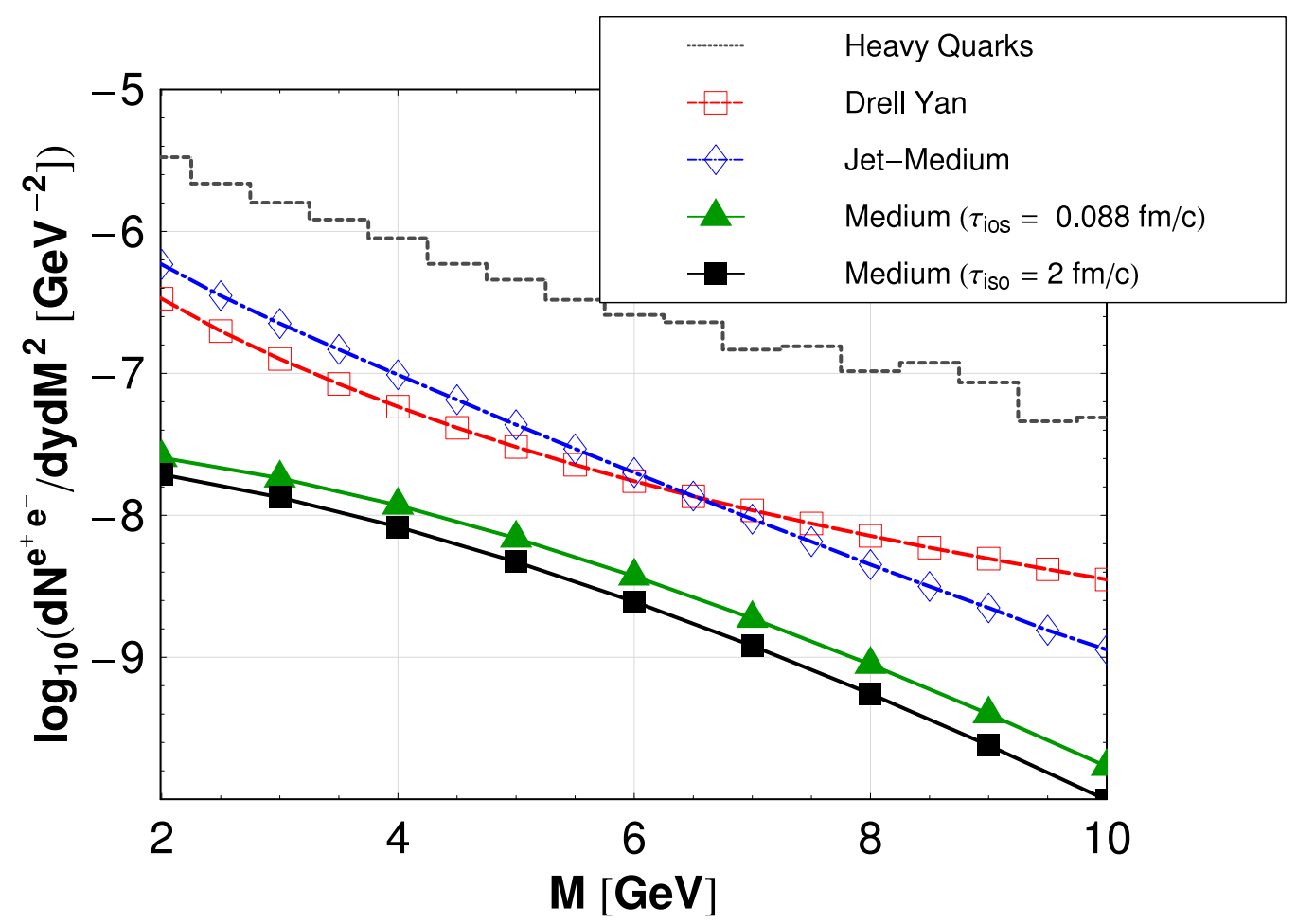

Figure 3: Collisionally-broadened interpolating model dilepton yields as a function of invariant mass in central $\mathrm{Pb}+\mathrm{Pb}$ collisions at the LHC, with a cut $p_{T} \geq 8 \mathrm{GeV}$ and and rapidity $y=0$. For medium dileptons we use $\gamma=2$ and $\tau_{\text {iso }}=0.088 \mathrm{fm} / \mathrm{c}$ for LHC energies and fixed final multiplicity. A $K$-factor of 1.5 was applied to account for NLO corrections. Dilepton yields from Drell Yan, Heavy Quarks, Jet-Thermal and Jet-Fragmentation were obtained from Ref. [18].

(Drell-Yan, jet-thermal, and jet-fragmentation). This coupled with the large background coming from semileptonic heavy quarks decays would make it extremely difficult for experimentalists to extract a clean medium dilepton signal from the invariant mass spectrum. For this reason it does not look very promising to determine plasma initial conditions from the dilepton invariant mass spectrum. Nevertheless, the situation looks better for the dilepton spectrum as a function of the transverse momentum as it is shown in Fig. 4. In this spectrum, there is an enhancemment of medium dilepton yield for $2<p_{T}<8 \mathrm{GeV}$ compared with the other sources of dileptons (Drell Yann and Jet conversion). Therefore, this observable offers the oportunity to have a cleanest way to determine plasma initial conditions.

In order to quantify the effect of time-dependent pre-equilibrium emissions we define the "dilepton enhancement", $\phi\left(\tau_{\text {iso }}\right)$, as the ratio of the dilepton yield obtained with an isotropization time of $\tau_{\text {iso }}$ to that obtained from an instantaneously thermalized plasma undergoing only $1+1$ hydrodynamical expansion, ie. $\tau_{\text {iso }}=\tau_{0}$.

$$
\phi\left(\tau_{\text {iso }}\right) \equiv\left(\frac{d N^{e^{+} e^{-}}\left(\tau_{\text {iso }}\right)}{d y d p_{T}^{2}}\right) /\left(\frac{d N^{e^{+} e^{-}}\left(\tau_{\text {iso }}=\tau_{0}\right)}{d y d p_{T}^{2}}\right) .
$$

This ratio measures how large the effect of early-time momentum anisotropies are on medium dilepton production. In the case of instantaneous isotropization, $\Phi\left(\tau_{\text {iso }}\right)$ is unity, and for $\tau_{\text {iso }}>$ 


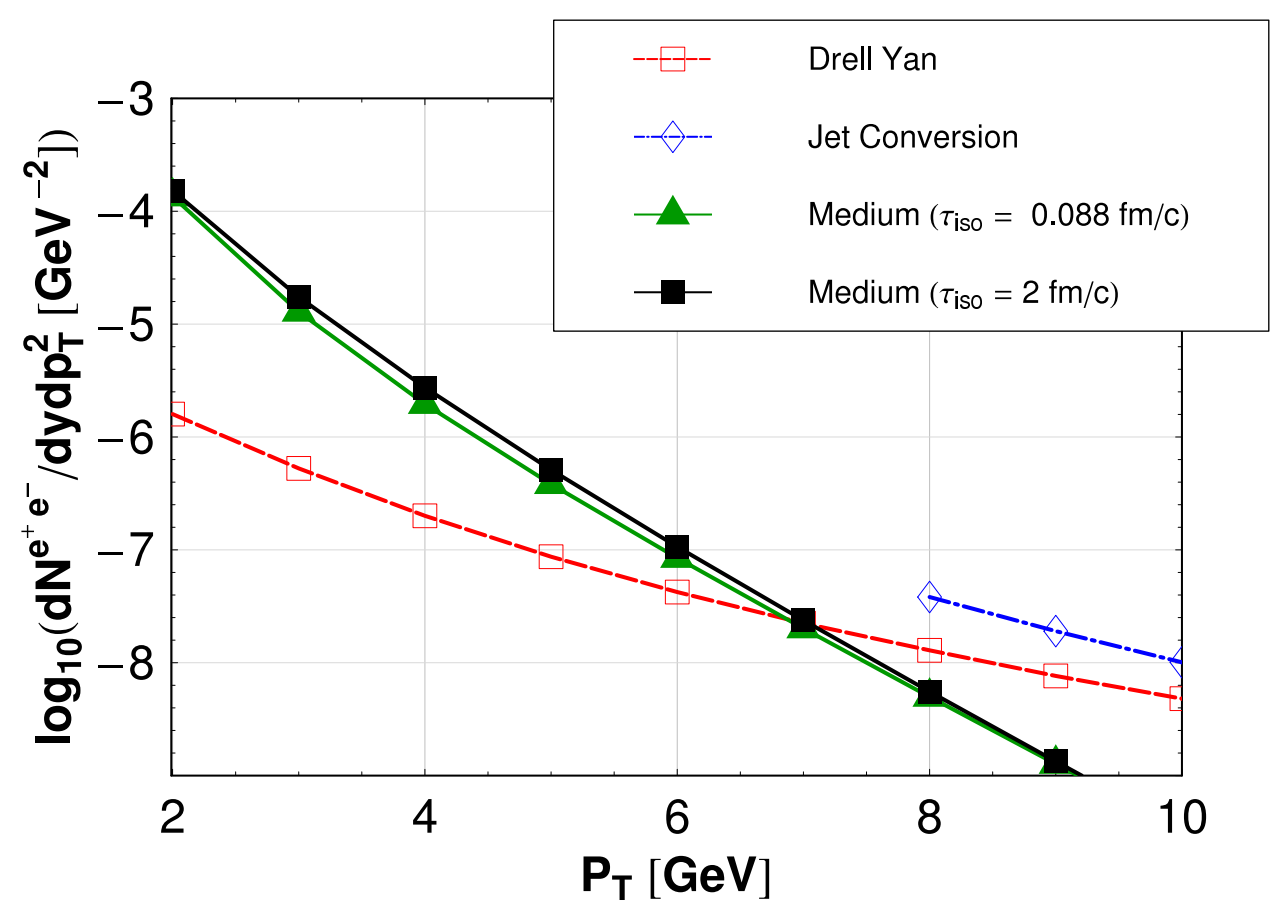

Figure 4: Collisionally-broadened interpolating model dilepton yields as a function of transverse momentum in central $\mathrm{Pb}+\mathrm{Pb}$ collisions at the $\mathrm{LHC}$, with a cut $0.5 \leq M \leq 1 \mathrm{GeV}$ and rapidity $y=0$. For medium dileptons we use $\gamma=2$ and $\tau_{\text {iso }}=0.088 \mathrm{fm} / \mathrm{c}$ for LHC energies and fixed final multiplicity. A $K$-factor of 6 was applied to account for NLO corrections. Dilepton yields from Drell Yan, Jet-Thermal and Jet-Fragmentation were obtained from Ref. [18].

$\tau_{0}$ any deviation from unity indicates a modification of medium dilepton production due to preequilibrium emissions.

In Fig. 5 we show the dilepton enhancement, $\phi$, as function of transverse momentum for $\tau_{\text {iso }}=$ $2 \mathrm{fm} / \mathrm{c}$ at LHC energies. The invariant mass cut is the same as in Fig. $4(0.5 \leq M \leq 1 \mathrm{GeV})$. As can be seen from Fig. 5, there is a rapid increase in $\phi 1$ and $4 \mathrm{GeV}$ at LHC energies. Moreover, from this figure both sharp and smooth transitions from early-time collisionally-broadened expansion to ideal hydrodynamic expansion result in a $30-50 \%$ at LHC energies. The effect of reducing $\tau_{\text {iso }}$ is to shift the peak in $\phi$ to larger $p_{T}$ while at the same time reducing the overall amplitude of the peak. Therefore, in order to see the difference between an instantaneously thermalized QGP with $\tau_{\text {iso }}=\tau_{0}$ and one with a later thermalization time requires determining the medium dilepton spectra between $2<p_{T}<8 \mathrm{GeV}$ at LHC with high precision so that one could measure the less than $50 \%$ variation resulting from pre-equilibrium emissions. Finally, we point out that it is possible to take other cuts (invariant mass and/or transverse momentum). This could be coupled with fits to experimental data, allowing one to fix $\tau_{\text {iso }}$ and $\gamma$ via a "multiresolution" analysis.

\section{Conclusions}

In this work we have introduced a phenomenological model that takes into account early-time momentum-space anisotropies in the rapidity dependence of high-energy dilepton production. To 


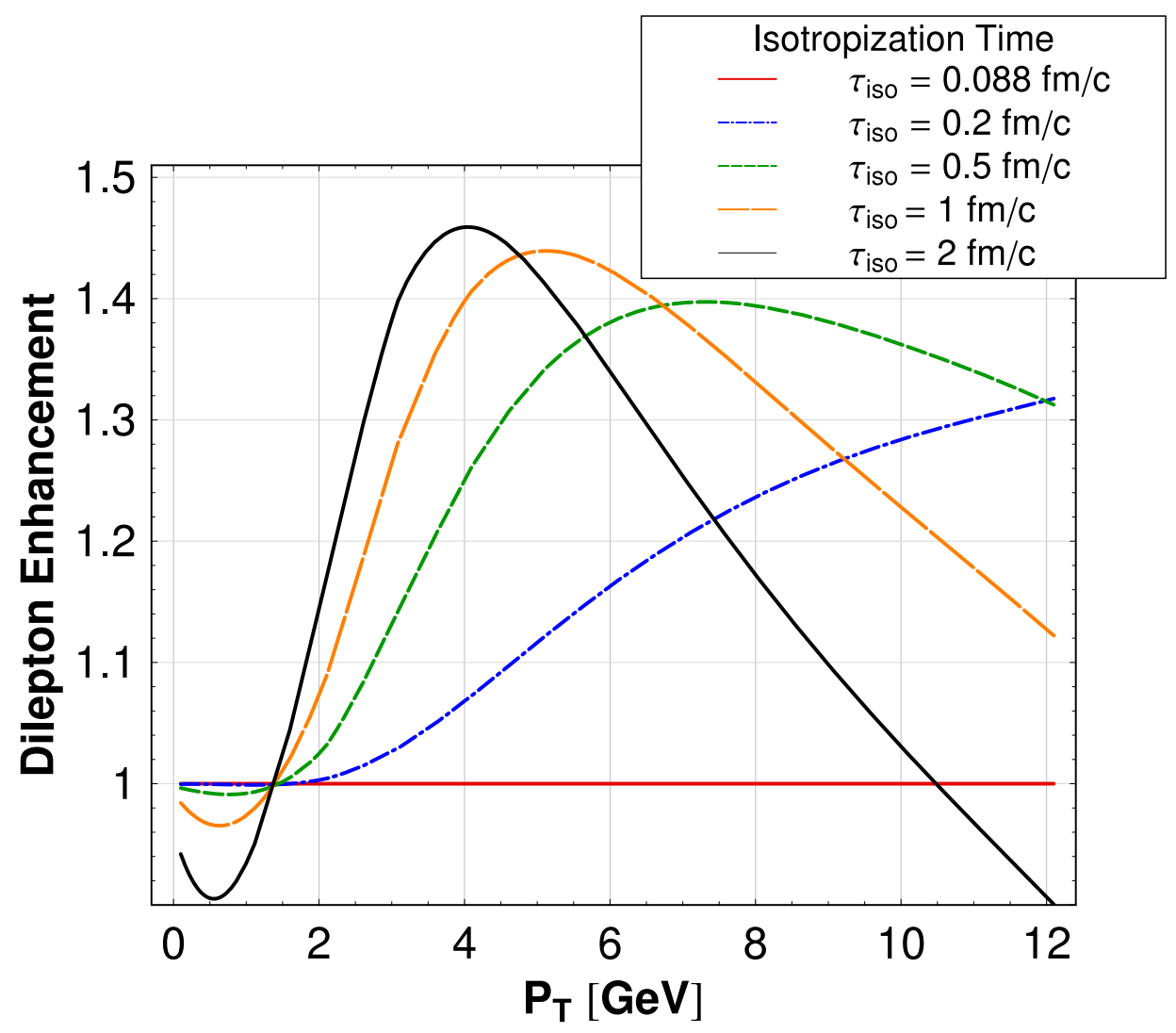

Figure 5: Dilepton enhancement, $\phi$ as defined in Eq. (4.1) resulting from our collisionally-broadened interpolating model with fixed final multiplicity and $\gamma=2$. The invariant mass cut used was $0.5<M<1$ $\mathrm{GeV}$, rapidity $y=0$ and $\tau_{0}=0.088 \mathrm{fm} / \mathrm{c}$ for LHC energies. Lines show expected pre-equilibrium dilepton enhancements for different values of the assumed plasma isotropization time, $\tau_{\text {iso }}$.

do this we have modeled the temporal evolution of the plasma anisotropy parameter $\xi$ and the hard momentum scale $p_{\text {hard }}$. Using these models we integrated the leading order rate for dilepton production in an anisotropic plasma over our modeled space-time evolution. Based on our numerical results for the variation of dilepton yields with the assumed values of $\tau_{\text {iso }}$, we find that the best opportunity to determine information about the plasma isotropization time is by analyzing the high transverse momentum $\left(2<p_{T}<8 \mathrm{GeV}\right.$ at LHC) dilepton spectra using relatively low pair invariant mass cuts $(M \lesssim 2 \mathrm{GeV})$. Based on these $p_{T}$ spectra we introduced the "dilepton enhancement" factor $\phi\left(\tau_{\text {iso }}\right)$ which measures the ratio of yields obtained from a plasma which isotropizes at $\tau_{\text {iso }}$ to one which isotropizes at the formation time, $\tau_{0}$. We find that there is a $30-50 \%$ enhancement in the high-transverse momentum dileptons at LHC energies when one assumes an isotropization time of $\tau_{\text {iso }}=2 \mathrm{fm} / \mathrm{c}$. The amplitude of the enhancement and position of the peak in the enhancement function, $\phi$, varies with the assumed value of $\tau_{\text {iso }}$ which, given sufficiently precise data, would provide a way to determine the plasma isotropization time experimentally. Another interesting possibility to constrain the value to $\tau_{\text {iso }}$ using dileptons is studying the forward region, where the effect of early-time anisotropies is expected to be maximal [27].

As an extension of the interpolating model presented in this work, one can consider the case 
where, instead of having at late times $1+1$ ideal hydrodynamical expansion, the system is described during that stage by $1+1$ viscous hydrodynamical expansion. This is possible since one can relate the anisotropy parameter, $\xi$, with the anisotropy in momentum-space of the fluid generated by the difference of the pressures along the longitudinal and transverse plane due to viscous corrections [28].

An uncertainty of our treatment comes from our implicit assumption of chemical equilibrium. If the system is not in chemical equilibrium (too many gluons and/or too few quarks) early time quark chemical potentials, or fugacities, will affect the production of lepton pairs [10, 11]. However, to leading order, the quark and gluon fugacities will cancel between numerator and denominator in the dilepton suppresion factor, $\phi\left(\tau_{\text {iso }}\right)[11]$. We, therefore, expect that to good approximation one can factorize the effects of momentum space anisotropies and chemical non-equilibrium.

We note in closing that within this model it is possible to assess the phenomenological consequences of momentum-space anisotropies on other observables which are sensitive to early-time stages of the QGP, e.g. photon production, heavy-quark transport, jet-medium induced electromagnetic and gluonic radiation, etc.

\section{References}

[1] T. Hirano and K. Tsuda, Collective flow and two pion correlations from a relativistic hydrodynamic model with early chemical freeze out, Phys. Rev. C 66, 054905 (2002) [nucl-th / 0205043 ].

[2] M. J. Tannenbaum, Recent results in relativistic heavy ion collisions: From "a new state of matter" to "the perfect fluid”, Rept. Prog. Phys. 69, 2005 (2006) [nucl-ex/ 0603003 ].

[3] D. Teaney, J. Lauret and E. V. Shuryak, Flow at the SPS and RHIC as a quark gluon plasma signature, Phys. Rev. Lett. 86 (2001) 4783 [nucl-th/ 0011058 ].

[4] P. Huovinen, P. F. Kolb, U. W. Heinz, P. V. Ruuskanen and S. A. Voloshin, Radial and elliptic flow at RHIC: Further predictions, Phys. Lett. B 503 (2001) 58 [hep-ph/ 0101136$].$

[5] R. Baier, A. H. Mueller, D. Schiff and D. T. Son, Bottom-up' thermalization in heavy ion collisions, Phys. Lett. B 502 (2001) 51 [hep-ph/ 0009237 ].

[6] Z. Xu and C. Greiner, Thermalization of gluons in ultrarelativistic heavy ion collisions by including three-body interactions in a parton cascade, Phys. Rev. C 71 (2005) 064901 [hep-ph/ 0406278 ].

[7] M. Strickland, Thermalization and the chromo-Weibel instability, J. Phys. G 34 (2007) S429 [hep-ph/0701238].

[8] M. Luzum and P. Romatschke, Conformal Relativistic Viscous Hydrodynamics: Applications to RHIC results at $\sqrt{\left(s_{N N}\right)}=200 \mathrm{GeV}$, Phys. Rev. C 78, 034915 (2008) [arXiv:0804 . 4015 [nucl-th]].

[9] J. I. Kapusta, L. D. McLerran and D. Kumar Srivastava, Rates for dilepton production at RHIC and LHC between J / $\psi$ and $\Upsilon$ are big, Phys. Lett. B 283, 145 (1992).

[10] A. Dumitru, D. H. Rischke, T. Schonfeld, L. Winckelmann, H. Stoecker and W. Greiner, Suppression of dilepton production at finite baryon density, Phys. Rev. Lett. 70, 2860 (1993).

[11] M. Strickland, Thermal photons and dileptons from nonequilibrium quark - gluon plasma, Phys. Lett. $B$ 331, 245 (1994). 
[12] P. Romatschke and M. Strickland, Collective Modes of an Anisotropic Quark-Gluon Plasma, Phys. Rev. D 68, 036004 (2003) [hep-ph/ 0304092$].$

[13] M. Martinez and M. Strickland, Pre-equilibrium dilepton production from an anisotropic quark-gluon plasma, Phys. Rev. C 78, 034917 (2008) [arXiv:0805.4552 [hep-ph]].

[14] M. Martinez and M. Strickland, Measuring QGP thermalization time with dileptons, Phys. Rev. Lett. 100, 102301 (2008) [arXiv:0709.3576 [hep-ph]].

[15] M. H. Thoma and C. T. Traxler, Production of energetic dileptons with small invariant masses from the quark-gluon plasma, Phys. Rev. D 56 (1997) 198 [hep-ph/ 9701354 ].

[16] P. Arnold, G. D. Moore and L. G. Yaffe, Photon and gluon emission in relativistic plasmas, JHEP 0206 (2002) 030 [hep-ph/ 0204343$].$

[17] F. Arleo et al., Photon physics in heavy ion collisions at the LHC, hep-ph / 0311131.

[18] S. Turbide, C. Gale, D. K. Srivastava and R. J. Fries, High momentum dilepton production from jets in a quark gluon plasma, Phys. Rev. C 74 (2006) 014903 [hep-ph / 0601042 ].

[19] A. Rebhan, M. Strickland and M. Attems, Instabilities of an anisotropically expanding non-Abelian plasma: 1D+3V discretized hard-loop simulations, Phys. Rev. D 78, 045023 (2008) [arXiv:0802.1714 [hep-ph]].

[20] S. Mrowczynski and M. H. Thoma, Hard loop approach to anisotropic systems, Phys. Rev. D 62, 036011 (2000) [hep-ph/ 0001164 ].

[21] P. Arnold, J. Lenaghan and G. D. Moore, QCD plasma instabilities and bottom-up thermalization, JHEP 0308, 002 (2003) [hep-ph/ 0307325$].$

[22] P. Romatschke and M. Strickland, Collective modes of an anisotropic quark-gluon plasma. II, Phys. Rev. D 70, 116006 (2004) [hep-ph / 0406188 ].

[23] D. Bodeker, The impact of QCD plasma instabilities on bottom-up thermalization, JHEP 0510, 092 (2005) [hep-ph/0508223].

[24] P. Arnold and G. D. Moore, The turbulent spectrum created by non-Abelian plasma instabilities, Phys. Rev. D 73, 025013 (2006) [hep-ph/0509226].

[25] P. Arnold and G. D. Moore, Non-Abelian Plasma Instabilities for Extreme Anisotropy, Phys. Rev. D 76, 045009 (2007) [arXiv:0706.0490 [hep-ph]].

[26] B. Schenke, M. Strickland, C. Greiner and M. H. Thoma, A model of the effect of collisions on QCD plasma instabilities, Phys. Rev. D 73, 125004 (2006) [hep-ph/ 060302 9].

[27] M. Martinez and M. Strickland, Suppression of forward dilepton production from an anisotropic quark-gluon plasma, arXiv:0808.3969 [hep-ph].

[28] M. Martinez and M. Strickland, Constraining relativistic viscous hydrodynamical evolution, arXiv:0902.3834 [hep-ph]. 